\title{
Close approach of a cloud of particles around an oblate planet
}

\author{
Vivian M. Gomes ${ }^{1}$. Geraldo M. C. Oliveira ${ }^{2,3}$. \\ Antonio F. B. A. Prado ${ }^{3}$. Diogo M. Sanchez 3
}

Received: 30 April 2015 / Revised: 6 July 2015 / Accepted: 24 July 2015 / Published online: 8 August 2015 (C) SBMAC - Sociedade Brasileira de Matemática Aplicada e Computacional 2015

\begin{abstract}
The goal of the present paper is to study close approaches of a cloud of particles with an oblate planet, which means that there is a $J_{2}$ term in the gravitational potential of the planet. This cloud of particles is assumed to be created during the passage of a spacecraft by the periapsis of its orbit, by an explosion or any other disruptive event. The system is formed by two large bodies (Sun and planet), assumed to be in circular orbits around the center of mass of the system, and the cloud of particles. The particles that belong to the cloud make a close approach to the flat planet and then they are dispersed by the gravitational force of the planet. The motion is governed by the equations of motion given by the planar restricted circular three-body problem plus the effects of the oblateness of the planet. Jupiter is used for numerical simulations. The results show the differences between the behavior of the cloud after the passage, considering or not the effects of the oblateness of the planet. The results show that the oblateness of the planet is equivalent to an increase in the mass of the planet.
\end{abstract}

Keywords Astrodynamics · Close approaches · Orbital maneuver · Oblate planet

Mathematics Subject Classification 37N05 - 70F07 · 70F15

\section{Introduction}

Many space missions used close approach maneuvers to save fuel in the orbital maneuvers required to reach their goals. The Voyager mission is one of the most important and well

Communicated by Elbert E. N. Macau, Antônio Fernando Bertachini de Almeida Prado and Cristiano Fiorilo de Melo.

$\triangle$ Diogo M. Sanchez

diogo.sanchez@inpe.br

1 State University of São Paulo, FEG/UNESP, Guaratinguetá, Brazil

2 Federal Center for Technological Education of Minas Gerais, DELCOM/CEFET-MG, Contagem, Brazil

3 National Institute for Space Research, DMC/INPE, São José dos Campos, Brazil 
known (Kohlhase and Penzo 1977) of those missions and it used a series of close approaches with the giant planets of the Solar System to make a tour to those planets. This maneuver has many applications and many other uses are available in the literature. Several of them are based on the idea of the Voyager mission, so use close approaches to reach the outer planets of the Solar System or even comets (Farquhar and Dunham 1981; Byrnes and D'Amario 1982; D'Amario et al. 1982; D'Amario and Byrnes 1983; Longuski and Williams 1991; Swenson 1992; Strange and Longuski 2002). Another possibility that received attention is the technique of using Venus to go to Mars (Hollister and Prussing 1966; Striepe and Braun 1991). Although Venus is an interior planet, a spacecraft launched in the direction of the Sun can make a close approach with Venus and be redirect to Mars, saving fuel or helping the mission to have a better timing; therefore, reaching Mars close to the time for a Hohmann transfer back to the Earth. A three-dimensional close approach with Jupiter can be used to make a modification in the orbital plane of the spacecraft, a very expensive type of maneuver if made using a propulsion system, which can send the spacecraft to an orbital plane that is perpendicular to the ecliptic (Carvell 1986), so making possible the observation of the poles of the Sun, the goal of the mission. Another interesting application is the use of the Moon to design a sequence of trajectories in the Earth-Moon system that passes by different altitudes (Marsh and Howell 1988); therefore, allowing the spacecraft to make observations in a larger region of the space. The use of multiple close approaches can also be considered, with the goal of obtaining optimal trajectories for a spacecraft (D'Amario et al. 1979, 1981). A mission to the Sun using close approaches with the inner planets of the Solar System also have been studied in the literature (Sukhanov 1999). This study shows how the inner planets remove energy from the spacecraft using close approaches, helping the spacecraft to be captured by the Sun. The moons of Jupiter were also considered bodies for similar maneuvers, as shown in references (Heaton et al. 2002; Longman and Schneider 1970). A detailed description of this type of maneuver is available in Broucke (1988), where the basic principles are clearly explained. To show better the possibilities given by this type of maneuver, a general mapping of close approach trajectories around the Moon is shown in Prado and Broucke (1995a), from where it is possible to find solutions for several types of problems, like escape or captures of a spacecraft by the Earth using a passage near the Moon to reduce the fuel expenditure in the process. The inclusion of other forces in the close approach mechanism is also available in the literature, as shown in reference (Prado and Broucke 1995b), where the drag due to the atmosphere of the Earth is added to the problem for the case of a single particle; and reference (Gomes et al. 2013), which considers the same effects for the case of a cloud of particles. Studies combining close approaches with celestial bodies and the use of a lowthrust propulsive system to make the necessary orbital maneuvers for a given mission are also considered, as shown in references (Casalino et al. 1999; McConaghy et al. 2003). A more complex close approach may consider the use of a tether to help the maneuver. References (Penzo and Mayer 1986; Puig-Suari et al. 1995; Lanoix 1996; Thompson 1998; Lanoix and Misra 2000) studied this problem. The main idea is to link the spacecraft to a celestial body by a cable and use this effect to increase the rotation angle of the maneuver. This maneuver is usually called "Tethered Gravity Assisted Maneuver" and can be interesting in several problems. Regarding the effects in cloud of particles, there are also some other recent studies in the literature, all of them considering a spherical planet for the close approach. Reference (Gomes and Prado 2008) studied this problem generalizing this study to all the planets of the Solar System, and reference (Gomes and Prado 2010) studied the problem of the effects of the initial energy in the dispersion of the cloud.

In the present paper, the effects of the oblateness of the planet during a close approach between a cloud of particles and a planet are considered. The study is limited to planar motion, 
because this region is where the effects of the oblateness of the planet are maximized. The equations of motion are based in the planar restricted circular three-body problem (Szebehely 1967), with the extra force that comes from the oblateness of the planet (Sanchez et al. 2009; Brouwer and Clemence 1961). This oblateness changes the distribution of mass of the planet, increasing the mass in the region of the equator, so increasing the gravitational forces in the equatorial plane. This form of the planet not only adds more mass to the equatorial region, but it also generates a mass distribution with averaged distances to the particles that are smaller when compared to a perfect spherical planet.

The cloud of particles is assumed to be formed at the periapsis of the trajectory, by an explosion of a spacecraft or any other type of disruptive event. It means that the initial conditions are specified at the periapsis of the trajectory. From this point, every single particle is numerically integrated forward in time until they reach a distance that can be assumed to be far from the planet, to make possible to neglect the effects of the planet, so considering the system as a two-body Sun-particle. At this point it is possible to compute the velocity, energy and angular momentum of the particle with respect to the Sun after the close approach with the planet, for every single particle. The semi-major axis and the eccentricity of those particles can be computed from those quantities, so it is possible to know the distribution of the orbital elements of the whole cloud. The assumptions of the present research make the planet to be in a circular orbit around the Sun, but any type of orbit is available for the spacecraft (elliptic, circular or hyperbolic).

Therefore, the main goal of the present research is to measure the differences of the orbital elements distribution of the particles of the cloud when considering the oblateness of the planet, compared with the similar situation, but with the close approaches made around a perfect spherical planet.

\section{Mathematical model}

To get accurate results, it is important to develop and use a mathematical model that is able to describe the main characteristics of the system. The circular restricted three-body problem is an excellent mathematical model to be used in this type of study, because it allows both primaries to act all the time in the motion of the spacecraft. Based on this model, it is possible to write the most important features of the system, as well as to derive the spacecraft equations of motion (Szebehely 1967). This problem is better studied if the canonical system of units is used. To use this system, it is necessary to define some parameters that will be used as units of mass, distance and time. The unit of mass will be the total mass of the system, which means the mass of the Sun $\left(M_{1}\right)$ added by the mass of the planet $\left(M_{2}\right)$, since the particles are assumed to have negligible mass. In this canonical system, the mass of the planet (called here $M_{2}$ ) is given by Eq. (1) shown below.

$$
\mu=\frac{M_{2}}{M_{1}+M_{2}}
$$

It implies that the mass of $M_{1}$ is given by Eq. 2 shown below.

$$
(1-\mu)
$$

Next, it is necessary to define the unit of distances. Since the orbit of the planet is assumed to be circular, its distance from the Sun is constant and this value can be used as the unit of distance. After this definition, it is possible to derive the equations of motion of the particles. The first step is to define an inertial reference system where the origin is located at the center 
of mass of the two massive bodies. The $x$-axis is the line connecting $M_{1}$ and $M_{2}$ and the vertical axis is the line perpendicular to the $x$-axis. In this system, $M_{1}$ and $M_{2}$ have positions that are given by Eq. 3. The unit of time is defined such that the period of the motion of the planet around the Sun is $2 \pi$.

$$
\begin{aligned}
& \bar{x}_{1}=-\mu \cos v \\
& \bar{y}_{1}=-\mu \sin v \\
& \bar{x}_{2}=(1-\mu) \cos v \\
& \bar{y}_{2}=(1-\mu) \sin v
\end{aligned}
$$

where $v$ is the true anomaly of the planet. In this system, the equations of motion of the spacecraft are given by Eqs. $(4,5)$.

$$
\begin{aligned}
\ddot{\bar{x}} & =\frac{-(1-\mu)\left(\bar{x}-\bar{x}_{1}\right)}{r_{1}^{3}}-\frac{\mu\left(\bar{x}-\bar{x}_{2}\right)}{r_{2}^{3}} \\
\ddot{\bar{y}} & =\frac{-(1-\mu)\left(\bar{y}-\bar{y}_{1}\right)}{r_{1}^{3}}-\frac{\mu\left(\bar{y}-\bar{y}_{2}\right)}{r_{2}^{3}}
\end{aligned}
$$

where $r_{1}$ and $r_{2}$ are the distances from the spacecraft to the Sun and the planet, respectively, given by Eqs. $(6,7)$ :

$$
\begin{aligned}
& r_{1}^{2}=\left(\bar{x}-\bar{x}_{1}\right)^{2}+\left(\bar{y}-\bar{y}_{1}\right)^{2} \\
& r_{2}^{2}=\left(\bar{x}-\bar{x}_{2}\right)^{2}+\left(\bar{y}-\bar{y}_{2}\right)^{2}
\end{aligned}
$$

Taking into account the disturbance due to the oblateness $\left(J_{2}\right)$ of the planet, there is a new term in the potential energy, given by (Sanchez et al. 2009; Brouwer and Clemence 1961) Eq. (8):

$$
U_{J 2}=-\mu R^{2} J_{2}\left(-\frac{1}{2} \frac{1}{r^{3}}\right)
$$

where $R$ is the equatorial radius of the planet. Considering this new term, the equation of motion of the spacecraft has the additional term shown by Eq. (9):

$$
\ddot{r}=-\frac{3}{2} \mu R^{2} J_{2} \frac{1}{r^{5}}
$$

That can be expressed in terms of $x-y$ components by Eqs. $(10,11)$ :

$$
\begin{aligned}
& \ddot{x}=-\frac{3}{2} \mu R^{2} J_{2} \frac{\left(\bar{x}-\bar{x}_{2}\right)}{r_{2}^{5}} \\
& \ddot{y}=-\frac{3}{2} \mu R^{2} J_{2} \frac{\left(\bar{y}-\bar{y}_{2}\right)}{r_{2}^{5}}
\end{aligned}
$$

There is one more important equation that is needed in the present paper. It is the so-called "Jacobian integral" (Szebehely 1967), which is a constant of motion of the restricted threebody problem. It is not valid after the inclusion of the term due to the oblateness of the planet, but it is one of the parameters that defines a close approach trajectory. Its importance will be shown later in this paper, when defining the steps that have to be made to solve the problem. It is given by Eq. (12). In this equation $E$ is the energy of the particle, $C$ is the angular momentum of the particle, $r_{1}$ is the Sun-spacecraft distance, $r_{2}$ is the Jupiter-spacecraft distance and $\omega$ is the angular velocity of the Sun-Jupiter system, assumed to be one in the canonical system of units used here. 
Fig. 1 Geometry of the close approach of the particles with Jupiter

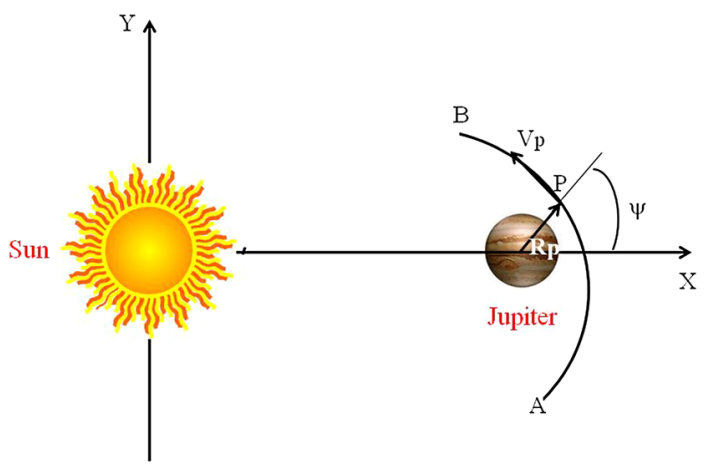

$$
J=E-\omega C=\frac{\dot{x}^{2}+\dot{y}^{2}}{2}-\frac{x^{2}+y^{2}}{2}-\frac{1-\mu}{r_{1}}-\frac{\mu}{r_{2}}
$$

The energy $(E)$ and angular momentum $(C)$ cited above are given by Eqs. (13) and (14) shown below, where $x, y$ and its time derivatives are the coordinates of the spacecraft measured in the rotating system.

$$
\begin{aligned}
& E=\frac{(x+\dot{y})^{2}+(\dot{x}-y)^{2}}{2}-\frac{1-\mu}{r_{1}}+\frac{\mu}{r_{2}} \\
& C=x^{2}+y^{2}+x \dot{y}-y \dot{x}
\end{aligned}
$$

Figure 1 describes the geometry of the problem. There is a spacecraft in a initial orbit around the Sun that is approaching Jupiter from point A. It enters the sphere of influence of Jupiter in a hyperbolic trajectory with respect to the planet and it reaches the periapsis of its orbit at the point $\mathrm{P}$, the point of minimum distance from Jupiter. This minimum distance is called $r_{p}$ and the angle between the periapsis line and the line Sun-Jupiter (horizontal axis) is represented by $\psi$ and it is called "angle of approach". The velocity of the spacecraft at this point is called "velocity at the periapsis" and it is represented by $V_{p}$. At this point it is assumed that the spacecraft becomes a cloud of particles, and every single particle follows its own trajectory.

\section{Parameters to specify one trajectory}

The first step to approach this problem is to choose the variables that describe the close approach trajectory. There are many alternatives. The following ones are used here (Broucke 1988):

(a) $\Psi$, the angle of approach, that defines the position of the periapsis of the hyperbolic trajectory of the spacecraft around Jupiter, as shown in Fig. 1;

(b) $J$, the Jacobian constant described in Eq. 12 that is used to specify the velocity of the spacecraft at the periapsis. It is used, instead of the velocity directly, to have results that can be easily compared with the literature (Broucke 1988; Prado and Broucke 1995a, b) that used this variable;

(c) $r_{p}$, the periapsis distance of the trajectory around Jupiter.

It is now necessary to specify the sequence of steps used to compare the trajectories generated by the models with and without the oblateness of the planet. They are: 
1. For the trajectory without oblateness, the numerical integration starts at the periapsis and proceeds until the point B (Fig. 1) is reached by the particle. The point B is defined to be the point where the distance Sun-spacecraft reaches 0.5 canonical units. At this point it is assumed that the Sun governs the motion of the spacecraft. The initial position is obtained from the variables $\Psi$ and $r_{p}$ and the initial velocity by the variables $\Psi$ and $V_{p}$. The Jacobian constant is specified and the velocity at the periapsis is obtained from there. The cloud of particles are created by adding small deviations to the nominal value of the variables $\Psi$ and $r_{p}$. It means that all the particles of the cloud have values for the angle of approach in the range of $\Psi \pm \delta \psi$ and periapsis distances in the range of $r_{p} \pm \delta r_{p}$. The Jacobian constant is assumed to be the same for all the particles, which means that the magnitude of their velocities are the same;

2. The trajectory including oblateness is similar. The numerical integration also starts at periapsis, keeping the same three parameters $\left(J, \Psi, r_{p}\right)$ of the previous maneuver. The only difference is that the term due to the oblateness of the planet (Eqs. 9-11) is added to the dynamics.

\section{Results}

To obtain a first view of the influence of the oblateness of the planet, two single trajectories are made using both models for the planet, spherical and oblate. Figure 2a shows the trajectories of a single particle obtained by assuming an oblate Jupiter, in green, and assuming a perfect spherical Jupiter, in red, for $\psi=333^{\circ}$ and $r_{p}=9100 \mathrm{~km}$ (1.3 radius of Jupiter). Figure $2 \mathrm{~b}$ shows the same type of trajectories, but for $\psi=180^{\circ}$ and $r_{p}=9100 \mathrm{~km}$. It is noted, in both of them, that the trajectories obtained by the oblate model have larger angles of curvature. This fact is compatible with the physical interpretation that the oblateness is equivalent to an increase in the mass of the planet, since the angle of curvature increases with the mass of the planet (Broucke 1988).

Next, it is important to concentrate in the main goal of the paper, which is measuring the effects of the oblateness of the planet in the distribution of the orbital elements of the particles of the cloud. To perform this task, the results are shown in plots having the semimajor axis of each particle in the horizontal axis and the eccentricity of each particle in the vertical axis, always after the passage by the planet. Three different values were used for the Jacobian constant $J:-1,0$ and 1 . This is equivalent to using three values for the velocity at the periapsis. Equation (12) indicates that increasing the value of the Jacobian constant increases the velocity of the particle in every point. Three values were also used for the angle of approach $\Psi: 0^{\circ}$, where there are no effects due to the close approach (Broucke 1988); $90^{\circ}$, where there is the maximum loss of energy due to the close approach (Broucke 1988); and $270^{\circ}$, where there is the maximum gain of energy due to the close approach (Broucke 1988). Figures 3, 4 and 5 show the results for the different parameters. Figure 3 shows the case where the Jacobian constant is -1 , Fig. 4 shows the case where it is 0 and Fig. 5 considers the situation where it is 1 . In all of those figures the black color represents the particles for the trajectories where the oblateness is included and the red color represents the situation where the planet is assumed to be a perfect sphere. The periapsis distance used was 10.000 $\mathrm{km}$ in all the cases.

There are many facts that can be observed from those results. The first one is the existence of open (hyperbolic) and closed (elliptical) orbits. It can be seen by remembering that open orbits have negative semi-major axis and eccentricities larger than one. In this aspect, it is 
$\mathbf{a}$ Trajectories of a single particle

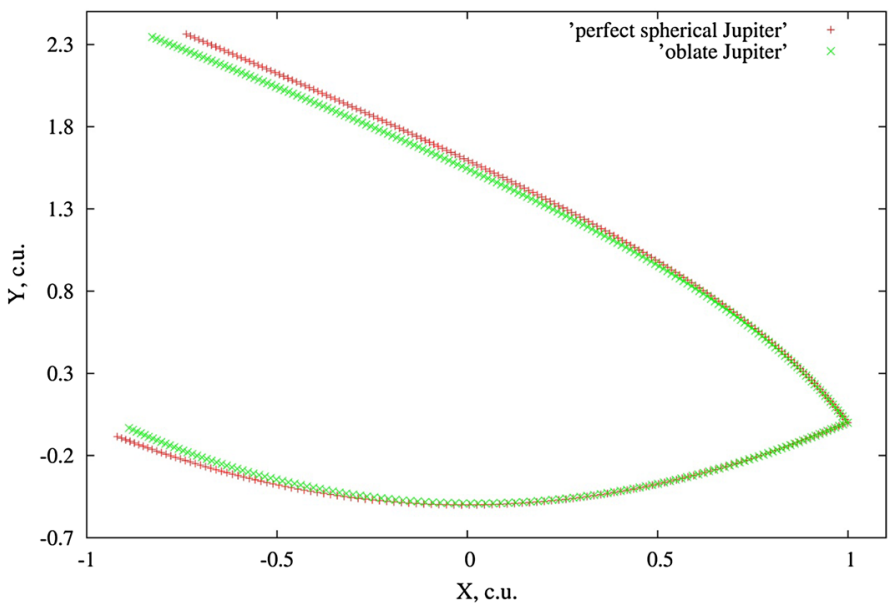

b

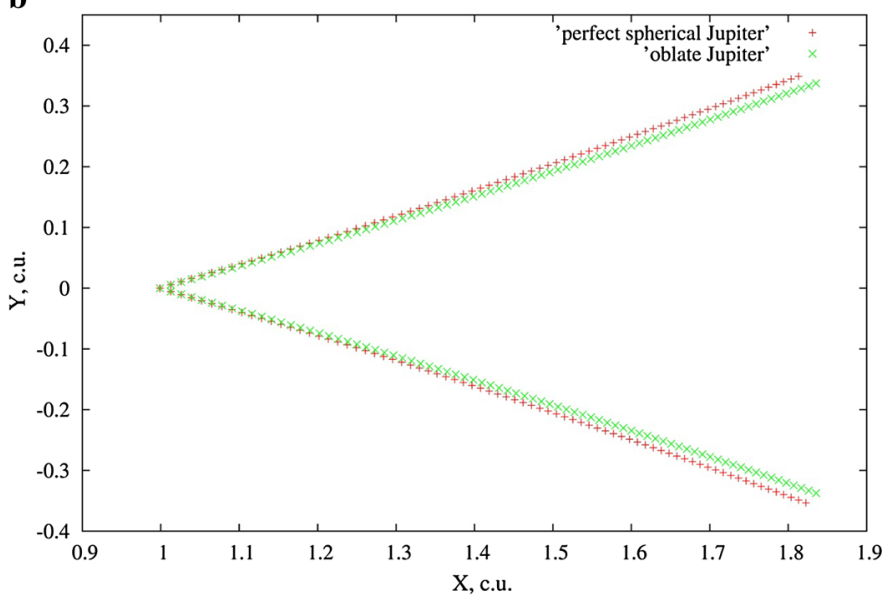

Fig. 2 a Trajectories of a single particle assuming an oblate Jupiter (green) and a perfect spherical Jupiter (red) for $\psi=333^{\circ}$ for $r_{p}=9100 \mathrm{~km}$ (1.3 radius of Jupiter). b Trajectories of a single particle assuming an oblate Jupiter (green) and a perfect spherical Jupiter (red) for $\psi=180^{\circ}$ for $r_{p}=9100 \mathrm{~km}(1.3$ radius of Jupiter)

also noted the expected dependence with the value of the Jacobian constant. For $J=-1$, the initial velocity is smaller, so only the situation where the angle of approach is $270^{\circ}$ has open orbits. In the situation where $J=0$ the same occurs, but when $J=1$ all the orbits are open. Besides that, it is noted that the open orbits have increasing semi-major axis when the value of the Jacobian constant gets higher.

The dependence with the angle of approach can also be explained by physical reasons. Passages with $\psi=90^{\circ}$ remove energy from the particle, and they finish the maneuvers with less energy. Close approaches with $\psi=270^{\circ}$ give extra energy to the particle, and they finish the maneuvers with more energy. The situation with $\psi=180^{\circ}$ does not modify the energy of the particle, and they finish the maneuvers with intermediate energy, when compared to the previous two cases. 

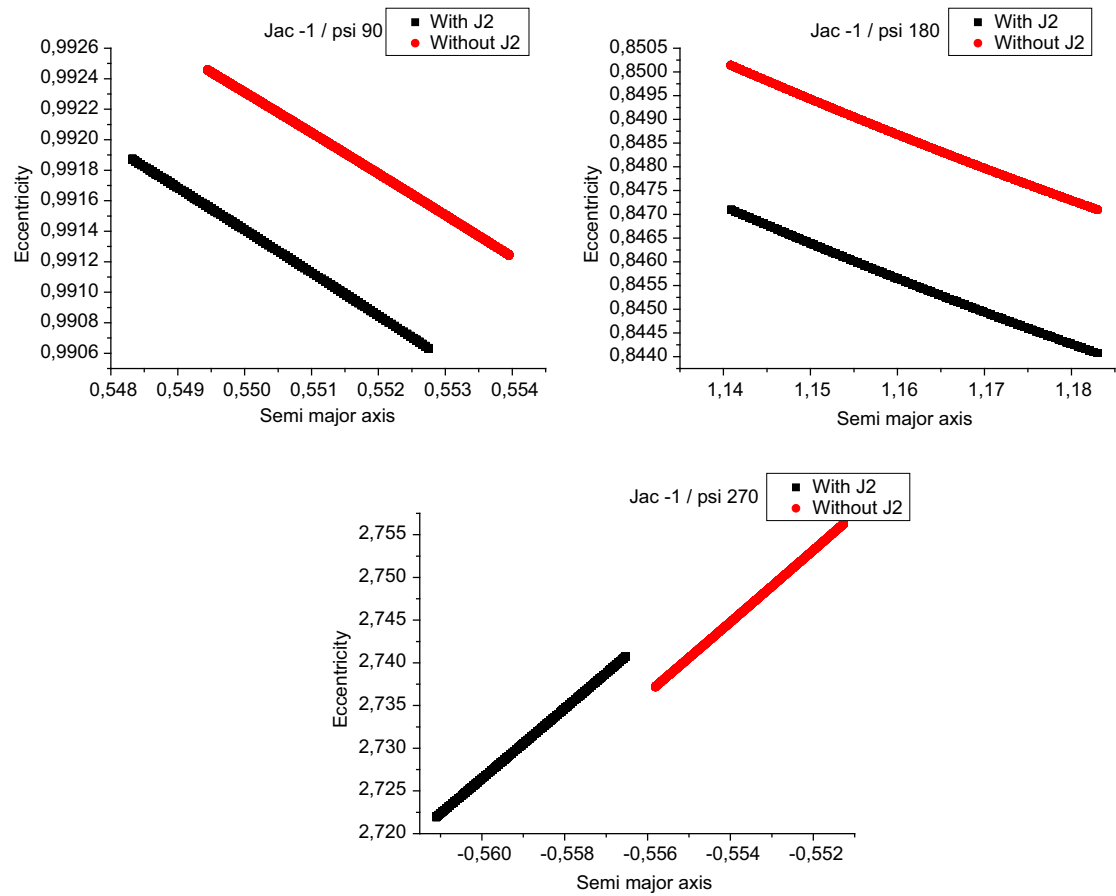

Fig. 3 Distribution of the orbital elements of the particles after the passage by the planet for $J=-1$
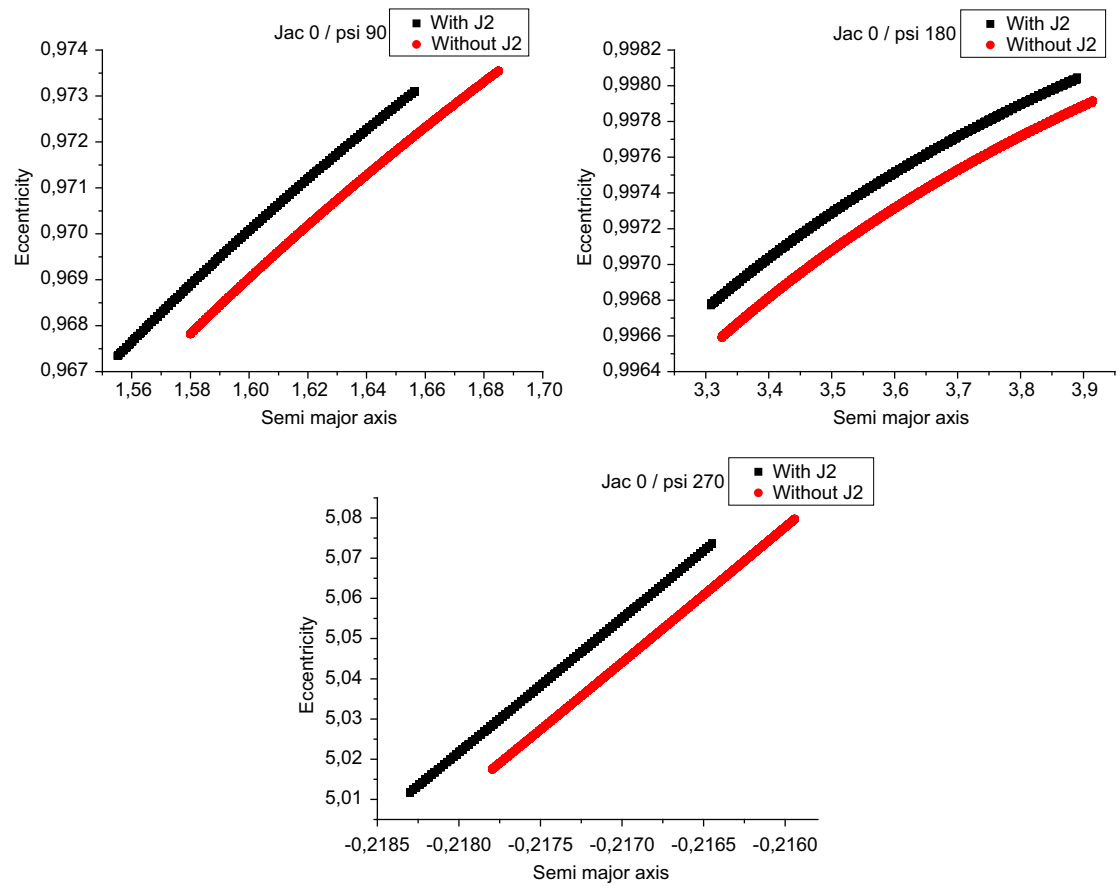

Fig. 4 Distribution of the orbital elements of the particles after the passage by the planet for $J=0$ 


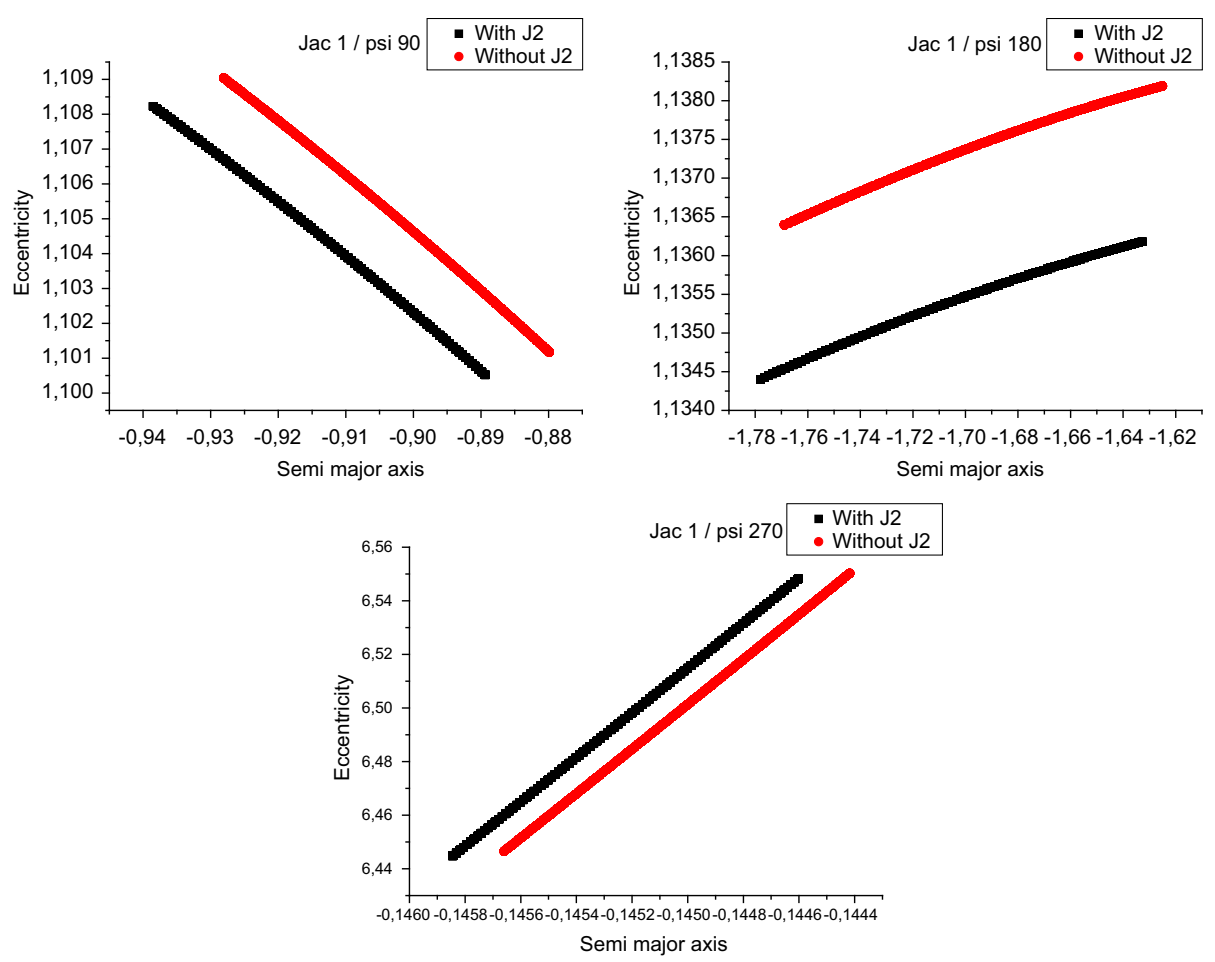

Fig. 5 Distribution of the orbital elements of the particles after the passage by the planet for $J=1$

It is also noted that the presence of the oblateness of the planet does not cause larger dispersions of the particles, because the cloud leaves the planet with similar dispersion. Note that both lines are nearly linear, parallel and with the same length in all the situations simulated. It means that the density of the cloud is not affected by the oblateness of the planet. The evolution of the density of the cloud is a very important point, if possible collisions with another spacecraft with this cloud is considered. The size of the cloud is also not modified, which is another important result from those simulations. The results also show that the inclusion of the oblateness causes an extra loss of energy, as expected, since the equivalent increase in the mass of the spacecraft takes more energy from the particle during the escape phase from the planet. This happens because the initial conditions, in particular the velocity, are specified at the periapsis. A planet with larger mass would first accelerate strongly the spacecraft during the incoming part of the trajectory, and then decelerate strongly during the exiting phase from the planet. It would make a zero balance effect, but, since the initial conditions are not specified at point $\mathrm{A}$, there is no compensation and the net balance is the reduction of the energy in all the situations, due to the extra deceleration during the phase where the spacecraft leaves the planet. This fact can be seen by looking at the extreme points of both lines, black and red. Every point in the black line always has a smaller energy when compared to the equivalent point of the red line, because the semi-major axis is always smaller. Remember that larger semi-major axis represents orbits with higher energy, since two-body dynamics are considered here. 


\section{Conclusions}

A numerical algorithm to calculate the effects of the inclusion of the oblateness of the planet in a close approach maneuver with Jupiter is developed. It is then used to study the behavior of the orbital elements of every single particle that belongs to a cloud of particles formed at the periapsis of the trajectory of the spacecraft. The results show several facts about this evolution. The oblateness of the planet acts like an increase in its mass, so reducing the energy of all the particles. The final result is that the distribution of the particles is affected by a general decrease in the energy level of the particles, but the density and size of the cloud of particles are not modified by this extra perturbation. This is an important result, if possible collisions with this cloud with another spacecraft have to be avoided.

Acknowledgments The authors wish to express their appreciation for the support provided by Grants \# 473387/2012-3,304700/2009-6 and 473164/2013-2 from the National Council for Scientific and Technological Development (CNPq); Grants \# 2011/08171-3, 2011/13101-4, 2014/06688-7, 2012/21023-6 and 2014/222955 from São Paulo Research Foundation (FAPESP) and the financial support from the National Council for the Improvement of Higher Education (CAPES).

\section{References}

Broucke RA (1988) The celestial mechanics of gravity assist. AIAA paper 88-4220. In: AIAA/AAS astrodynamics conference, Minneapolis, MN, 15-17 Aug

Brouwer D, Clemence G (1961) Methods of celestial mechanics. Academic Press, Massachusetts

Byrnes DV, D’Amario LA (1982) A combined Halley flyby Galileo mission. AIAA paper, 82-1462

Carvell R (1986) Ulysses-the Sun from above and below. Space 1:18

Casalino L, Colasurdo G, Pastrone D (1999) Optimal low-thrust escape trajectories using gravity assist. J Guidance Control Dyn 22(5):637-642

D’Amario LA, Byrnes DV (1983) Interplanetary trajectory design for the Galileo mission. In: AIAA, aerospace sciences meeting, vol 1

D’Amario LA, Sackett LL, Stanford RH, Byrnes DV (1979) Optimization of multiple flyby trajectories. In: American Institute of Aeronautics and Astronautics conference, vol 1

D’Amario LA, Byrnes DV, Stanford RH (1981) A new method for optimizing multiple-flyby trajectories. J Guidance Control Dyn 4(5):591-596

D'Amario LA, Byrnes DV, Stanford RH (1982) Interplanetary trajectory optimization with application to Galileo. J Guidance Control Dyn 5(5):465-471

Farquhar RW, Dunham DW (1981) A new trajectory concept for exploring the Earth's geomagnetic tail. J Guidance Control Dyn 4(2):192-196

Gomes VM, Prado AFBA (2008) Swing-by maneuvers for a cloud of particles with planets of the solar system. WSEAS Trans Appl Theor Mech 3(11):869-878

Gomes VM, Prado AFBA (2010) A study of the impact of the initial energy in a close approach of a cloud of particles. WSEAS Trans Math 9(10):811-820

Gomes VM, Prado AFBDA, Golebiewska J (2013) Dynamics of space particles and spacecrafts passing by the atmosphere of the Earth. Sci World J 2013:1-6

Heaton AF, Strange NJ, Longuski JM, Bonfiglio EP (2002) Automated design of the Europa Orbiter tour. J Spacecr Rockets 39(1):17-22

Hollister WM, Prussing JE (1966) Optimum transfer to Mars via Venus (transfer paths to Mars via Venus compared with direct flight, discussing direct free fall transfer, flyby and free fall transfer). Astronaut Acta 12:169-179

Kohlhase CE, Penzo PA (1977) Voyager mission description. Space Sci Rrev 21(2):77-101

Lanoix E (1996) Tether sling shot assists-a novel approach to travelling in the solar system. In: CASI conference on astronautics - towards the next century in space, 9th, Ottawa, Canada, pp 62-71

Lanoix ELM, Misra AK (2000) Near-earth asteroid missions using tether sling shot assist. J Spacecr Rockets 37(4):475-480

Longman RW, Schneider AM (1970) Use of Jupiter's moons for gravity assist. J Spacecr Rockets 7(5):570-576

Longuski JM, Williams SN (1991) The last grand tour opportunity to Pluto. J Astronaut Sci 39:359-365 
Marsh SM, Howell KC (1988) Double lunar swingby trajectory design. AIAA paper, 88-4289

McConaghy TT, Debban TJ, Petropoulos AE, Longuski JM (2003) Design and optimization of low-thrust trajectories with gravity assists. J Spacecr Rockets 40(3):380-387

Penzo PA, Mayer HL (1986) Tethers and asteroids for artificial gravity assist in the solar system. J Spacecr Rockets 23(1):79-82

Prado AFBA, Broucke R (1995a) Classification of swing-by trajectories using the Moon. Appl Mech Rev 48(11S):S138-S142

Prado AFBA, Broucke R (1995b) Effects of atmospheric drag in swing-by trajectory. Acta Astronaut 36(6):285-290

Puig-Suari J, Longuski JM, Tragesser SG (1995) A tether sling for lunar and interplanetary exploration. Acta Astronaut 35:671-680

Sanchez DM, Yokoyama T, Brasil PIDO, Cordeiro RR (2009) Some initial conditions for disposed satellites of the systems GPS and Galileo constellations. Math Probl Eng 2009:510759. doi:10.1155/2009/510759

Strange NJ, Longuski JM (2002) Graphical method for gravity-assist trajectory design. J Spacecr Rockets 39(1):9-16

Striepe SA, Braun RD (1991) Effects of a Venus swingby periapsis burn during an Earth-Mars trajectory. J Astronaut Sci 39(3):299-312

Sukhanov AA (1999) Close approach to Sun using gravity assists of the inner planets. Acta Astronaut 45(4):177-185

Swenson BL (1992) Neptune atmospheric probe mission. AIAA paper (92-4371)

Szebehely VG (1967) Theory of orbits. Academic Press, New York

Thompson WB, Stern MO, Dubin DHE (1998) A skyhook from Phobos to Mars. J Br Interplanet Soc 51(3):99_ 115 